bestimmung: einfaches 548, 25. 26. 568, 1; zusammengesetztes 544, 12 . 548, 26. 549, 14. 550, 21. 558, 10. 11. 560, 8. 10. 562, 22. 571, 17 . Objectiver genetiv beim infinitiv ist auch jüngerer sprachgebrauch; damit dürfte wahrscheinlich werden, dass XVII jünger ist als V. ' Die predigt I (vgl. s. 547) stimmt auch in der behandlung des infinitivs zu XVII: vgl. 6, 8. 9. 35 . 7, 10. 13. 24. 8, 27. $(10,7)$.

GIESSEN, 21. mai 1908.

OTTO BEHAGHEL.

\title{
ZUR DEUTSCHEN ETYMOLOGIE.
}

I.

'Zur deutschen etymologie' hat Uhlenbeck 1901, Beitr. 26. 290-312 einen aufsatz geschrieben, in dessen mittelpunkt eigentlich mein Etymologisches wörterbuch der dentschen sprache zumal in der 1898 vollendeten 6. auflage steht. Es ist etwas spät, dass ich jetzt zu diesem aufsatz das wort ergreife und meinen von Uhlenbeck angegriffenen standpunkt verteidige. Meine verteidigung fällt mir heute viel leichter, als sie mir wol vor 7 jahren gewesen wäre. Damals hätte ich die polemik im wesentlichen mit eigenen waffen führen müssen, jetzt habe ich ganż unerwartet einen bundesgenossen erhalten, der für mich streiten kann. Ich meine den neuen Weigand, von dem bis jetzt, october 1908, die vier ersten hefte (A-Grille) vorliegen.

Ueber die etymologische seite der neuen auflage von Weigands Wörterbuch hat Uhlenbeck - im hinblick widerum auf mein Etymolog. wörterbuch - soeben in einer besprechung des Museums 1908, spalte 37 folgende äusserung: getan: 'In elk geval moet worden erkend, dat wij thans weer een goed etymologisch woordenboek van het Duitsch bezitten, dat op de hogte van den tijd is, iets wat sedert lang niet.meer van Kluge's werk mag gezegd worden.' Dieses urteil Uhlenbecks berechtigt mich, den etymologen des neuen Weigand mit der mehrzahl der einwendungen zu confrontieren, die Uhlenbeck Beitr. 26, 290 ff. gegen mich erhoben hat. 
1. Aalraupe. Ich bezeichne verwantschaft mit aksl. ryba als unsicher; Uhlenbeck hält sie für sicher; aber der neue Weigand hält sie nicht einmal für erwähnungswert.

2. Anger. $\mathrm{Zu}$ diesem artikel trägt $U$. mnl. enck (dat. enghe) m. 'grasland', nnl. dial. eng 'ackerland' nach und erwähnt als verwant aind. anc- 'biegen'. Hirt scheint das abzulelnen, jedenfalls erwähnt er es nicht.

3. Bach. Uhlenbeck sagt, dass ich mich der jüngeren etymologischen forschung gegenüber zu ablehnend verhalte, und begründet dies damit: "warum erwähnt Kl. z. b. mit keinem worte die von Kern (Tijdschr. v. ned. taal- en letterk. 4, 316) herrührende etymologie von bach (: aind. bhaj- 'brechen')?" (s. 291). Mittlerweile wird sich U., der ja die neue auflage von Weigand besprochen hat, überzeugt haben, dass der neue Weigand diese erklärung keines wortes würdigt. Oder verhält sich auch der nene Weigand der jüngeren etymologischen wortforschung gegenüber $\mathrm{zu}$ ablehnend?

4. Beere. "Die evident richtige erklärung von beere als ableitung von norw. bas, base 'kleines gebüsch' (Bugge, Beitr. 21, 421) scheint Kl. nicht überzeugt zu haben" (s. 292). - Aber den neuen Weigand auch nicht, denn sonst hätte der sie wol erwähnt.

5. Blöde. „Nach $\mathrm{Kl}$. hat blöde keine verwanten ausserhalb des germ., obwol der vergleich von gr. 4 2avoos doch sehr nahe liegt" (s. 292). Von dieser so naheliegenden griechischen verwantschaft schweigt aber auch der neue Weigand.

6. Bohren. "Mit bohren ist zwar nicht aind. Zhurij- zu vergleichen, sondern vielmehr aind. blurinäti 'versehrt', avest. 3. pl. brīnanti, np. bur(r)ĩdan 'schneiden', aksl. briti 'scheren' " (s. 292). Hirt vergleicht natiurlich aind. bhurij-, aber nicht aind. bhrinati, auch nicht die pers. und slavischen worte. Der denkende leser kommt allmählich auf den verdacht, dass nicht bloss ich mit meinem Etymolog. wb., sondern auch der neue Weigand mit seinen etymologien sich den neneren etymologischen forschungen gegenüber $z u$ ablehnend verhält.

7. Braut. "Bei braut finden wir wol lat. Frutis, nichts aber über möglichen zusammenhang mit aind. bru-, avest. mru-" (8. 292). Selbstredend hält anch Hirt diese deutung nicht für erwähnenswert. 
8. Brunnen. Gegenüber meinem artikel fragt U., „ob wir brennen von air. brennim 'sprudeln' trennen dürfen?" (s. 293). Hirt würdigt auch diese etymologie keines wortes, aber U. macht ihm in seiner vorhin citierten besprechung keinen vorwurf, so dass man den verdacht wagen darf, $U$. würdige sie selber nicht mehr der erwähnung. Oder soll ich etwa glauben, dass U. meine leistungen mit einem anderen masse misst als den netien Weigand?

9. Bulle „könnte ursprünglich 'grosses, starkes tier' bedeutet haben und zur indog. wz. *bheū $\bar{\alpha}$ - 'wachsen' $=* b h e u \bar{a}$ "werden' gehören" (s. 293). Im Weigand erfährt man davon kein wort, dass bulle eigentlich 'der grosse' bedeutet habe. Aber der neue Weigand nimmt in der etymologie entschieden einen modernen standpunkt ein, und mein wörterbuch zeigt natürlich spuren des veraltens, wenn ich bullie nicht als 'den' grossen' deute.

10. Butte „harrt noch immer der erklärung. Das daneben stehende starke masculinum mnl. but, bot ist wol nicht ursprünglich. Ich gehe aus von urgerm. *buttonn- (woneben vielleicht *buttjon- wegen butte neben botte) und stelle dies mit $t t$ aus dhn zu indog. *bhudhnó- 'boden, grund, meeresgrund' (aind. budhná- u.s.w.). Vorgerm. *bhudhn(i) $\bar{o} n$ - ist also eigentlich 'zum meeresgrunde gehörig, in der meerestiefe lebend' " (s. 293). Diese neue deutung, die ich bei der 6. aufl. meines Et. wb. nicht benutzen konnte, übergeht Hirt selbstverständlich mit stillschweigen.

11. Drohen. „Ahd. drouwen, ags. préazean 'drohen', ahd. drō, ags. préa 'drohung' sind kaum von russ. traviť, poln. trawić 'hetzen, jagen' zu trennen (indog. wz: *trāu-). Meines wissens hat niemand diese gleichung aufgestellt" (s. 294). Weigand, der sich der neuen etymologischen forschung gegenüber nicht so ablebnend verhält wie ich, lehnt auch diese neue etymologie ab, ohne ein wort darüber zu verlieren.

12. Duft. „Jedenfalls gehört duft, wie schon. Persson (Wurzelerw. 285) angenommen hat, zu einer labialerweiterung

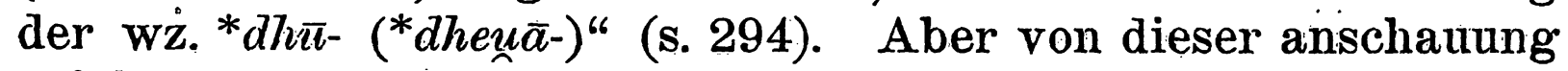
erfährt man auch im neuen Weigand nichts.

13. Düster. Das germ. wort „scheint nach $\mathrm{Kl}$. vereinzelt dazustehen" (s. 294). Nun vergleicht U. russ. tusł.lyj 'dunkel, 
trübe' und serb. natuštiti se, stuštiti se 'sich verfinstern'. Aber auch diese deutung straft der neue Weigand mit geringschätzung.

14. Eis. Uhlenbeck äussert sich darüber (s. 294): „ich deute germ. *isa n. (m.) 'eis' als 'das schlüpfrige' und stelle es $\mathrm{zu}$ aind. éshati 'schleicht, gleitet'." Unser etymologe hat entschieden pech mit seinen neuen etymologien, nicht einmal freund Hirt glaubt daran. Wie kann er auf anerkennung von mir rechnen, der sich bekanntlich den neueren etymologischen forschungen gegenüber zu ablehnend verhält?

15. Feme. Widerum trägt $U$. eine neue deutung vor, ohne den beifall des modernen etymologen zu gewinnen, der darüber kein wort verlieren zu müssen glaubt!

16. Fessel. „Eine spur von germ. *fatila- $=$ hd. fessel glaube ich im slavischen nachweisen zu können: vgl. aksl. petlja 'band, gehenk', das sich als entlehnung aus einer niederdeutschen form mit umlaut (etwa *fetilja f.) erklären lässt" (s. 296). Auch das fehlt bei Weigand.

17. Flied er. „Mnd.vlēder, as. *fliodar, *fliothar, nl. vlie(de)r (Kluge 6 117) kann mit lit. putinas 'wasserhollunder' zusammenhängen, falls dieses unter dem einfluss von púti 'faulen' an die stelle von *plutinas getreten ist" (s. 297). Auch diese neue deutung findet bei Hirt nur misachtung; er sagt schlankweg 'dunklen ursprungs'. Ich hatte freilich gesagt 'ursprung dunkel'.

18. Geck. Eine neue erklärung des wortes von U. s. 297 bleibt den lesern des neuen Weigand durchaus vorenthalten. Wir erfahren nur: "weitere herkunft unbekannt; vgl. aber Uhlenbeck, Beitr. 26, 290." Hirt hat gewiss die neue deutung nicht ernst genommen.

19. Gerte. "Trotz Kluge 142 hat ahd. gartea, gerta, ags. zerd, zierd, zyrd nichts mit got. gazds zu schaffen" (s. 298). Selbstredend hält Hirt die von mir vertretene etymologie für durchaus berechtigt und straft sie nicht mit derselben geringschätzung, mit der er Uhlenbeck'sche etymologien nun so oft schon behandelt hat.

\section{II.}

Uhlenbecks aufsatz (Beitr. 26, $290 \mathrm{ff}$.) hat auf 20 seiten. mein Etymologisches wörterbuch zum gegenstand der kritik gemacht. Ich habe mich im vorstehenden meiner eigenen 
meinungen durchaus enthalten und habe einen etymologen, der gewiss nicht parteiisch für mich eintreten wird, zum unparteiischen über Uhl.'s einwände gegen mich gemacht. Soweit der neue Weigand jetzt vorliegt, habe ich auf den vorstehenden 4 seiten die ersten 8 seiten von U.'s einwänden in Hirt'scher beleuchtung gezeigt. Wenn nun $U$. in seiner besprechung des neuen Weigand (Museum 1908, sp. 371) es wider für der mühe wert hält, mein wörterbuch als veraltet hinzustellen und zu erklären, dass uns im neuen Weigand endlich wider einmal ein auf der höhe der etymologie stehendes werk beschert ist, so hat der neue Weigand für mich und jeden vorurteilsfreien bewiesen, wie ernsthaft Uhlenbeck'sche etymologien zu nehmen sind. Es hätte gar keinen zweck, nun auch meinerseits mit U. zu rechten, nachdem der sprachvergleicher Hirt sein ablehnendes verhalten U̇. gegenüber offenkundig zur schau getragen hat. Es hat umso weniger zweck, als unsere beiderseitigen standpunkte viel zu verschieden sind, als dass eine einigung zu erhoffen wäre.

Erstens vertragen sich unsere grammatischen anschauungen nicht miteinander. So führt U. (Beitr. 26, 309) den inneren dental von ahd. stüda auf indogerm. $d h$ - zurück, und kommt damit zu der neuen annahme von verwantschaft mit aksl.

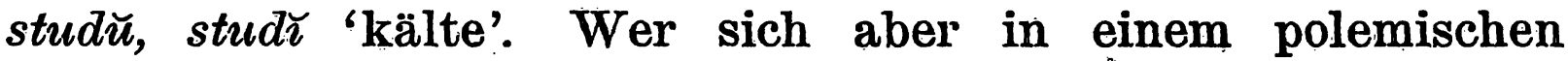
artikel an deutsche wortforscher heranwagt, miisste eigentlich doch wol wissen, dass das inlautende $d$ des ahd. auf urgerm. $\underline{p}$ $=$ indogerm. $t$ zurückgeht. Wer nicht einmal mit der hochdeutschen lautverschiebung ordentlich umgehen kann, der sollte doch lieber das gebiet der deutschen etymologie gar nicht betreten.

Beitr. 26, 300 erklärt U. das $f$ von schlittenkufe für germanisch, um mir daraufhin vorzuwerfen, dass ich es fälschlich mit lit. $\dot{z}$ aginys 'palisaden, pfosten' verbinde. Dass das $f$ von kufe wie das entsprechende ch des synonymen ahd. chuohha, wie es $U$. aus meinem Et. wb. übernimmt, eine gemeingermanische tenuis zur voraussetzung hat, das hätte U. wol wissen können, wenn er sich mit den etymologien hochdeutscher worte beschäftigt.

Aber auch mit der urgermanischen lautverschiebung steht unser etymologe in schlechten beziehungen: man braucht sich 
nur Beitr. 33, 184 die behandlung von ahd. itis anzusehen. Jeder anfänger auf dem gebiete der deutschen lautgeschichte weiss, dass dies nicht auf die von Uhlenbeck angenommene idg. grundform etesí- zurückgehen kann, eine solche grundform hätte naturgemäss zu germ. $z=$ westgerm. nord. $r$ führen müssen. Ein etymologe, der in germanistische zeitschriften schreibt, sollte doch eigentlich das Verner'sche gesetz handhaben können. Uebrigens dürfte bei deutung der grundform keineswegs die consonantische flexion von ahd. itis (= asächs. idis) ausser acht gelassen werden, die doch aller wahrscheinlichkeit nach älter ist, als zugehörige formen der $i$-declination. Vollends weiss U. nichts von einer ahd. nebenform itins, deren $n$ in den sächsischen dialekten lautgesetzlich geschwunden sein kann. Wenn man alle formellen schwierigkeiten, die das wort schon dem vorsichtigen Kögel (Beitr. 16, 502) bereitet hat, so unbefangen ignoriert wie U., ist man in der tat schnell mit einer schönen etymologie fertig. Dass ihm dabei in Leiden der aufsatz von v. Grienberger in der Zeitschrift für deutsche philologie nicht zur verfügung steht, erleichtert es ihm natürlich auch noch, mit den schwierigkeiten schnell fertig zu werden.

Noch ein beispiel, wie wenig U. mit der 1. lautverschiebung umgehen kann, ergibt seine behandlung von got. brüps (Beitr. 22, 188); wo er eine germ. grundform brüpi- ansetzt, ohne auch nur ein wörtchen darüber zu verlieren, dass wir von einer germ. grundform br·̄udi- ausgehen müssen. Erst seit Braunes artikel über das wort braut Beitr. 32, 30 (1907) wissen wir, dass wir vielleicht für das gotische eine solche grundform anzunehmen haben. Aber von der vorsichtigen beweisführung Braunes konnte $U$. nichts ahnen, und so war damals sein ansatz von germ. brüpi- ohne irgendwelche begründung sicher ein grober fehler.

Zweitens aber stellen wir beide an die glaubhaftigkeit von etymologien ganz verschiedene ansprüche. So werde ich jetzt (Beitr. 33, 104) aufmerksam auf. U.'s neue deutung von ahd. gouh 'kuckuck', worin die vorsilbe ga- und irgend eine nominalbildung zu germ. aukan 'vermehren' stecken soll: nach U. bedeutet ahd. gouh aus ga-aulc etymologisch eigentlich 'miternährter'. Diese Nordisk Arkiv 1.5, 151 veröffentlichte neue etymologie würdigt der neue Weigand unter dem worte yauch 
keiner erwähnung. Ich aber erwähne sie hier nur, um zu zeigen, dass zwischen $U$. und mir in etymologischen dingen nicht leicht eine einigung erzielt werden kann.

Aber gibt es überhaupt zwei forscher, deren anschauungen sich in etymologischen dingen vollständig decken? Es gibt jedenfalls eine ganże reihè von etymologien, deren glaubhaftigkeit und sicherheit bisher so fest stand, dass man zweifel für unmöglich hielt. Und doch hat neuerdings z. b. ein so ausgezeichneter forscher wie Pogatscher die gleichung ags. wer = lat. vir aufgehoben und für ags. wer 'mann' das altgerm. -varii in den völkernamen Amsivarii, Angrivarii als den nächsten verwanten behauptet. Müssen wir aber die gleichung ags. ver = lat. vir aufgeben, so müssen wir auch der mehrzahl aller neueren etymologien die berechtigung absprechen. Aber ich glaube, die meisten von uns werden sich nicht in der alten annahme beirren lassen. - Dass ahd. bibêt 'er bebt' skr. bibhêti 'fürchtet sich' ist und beide auf einer gemeinindog. wurzel $b \grave{h} i$ - 'sich fürchten, beben' beruhen, galt vielen von uns als ein dogma, aber Wackernagel, Kuhns zs. 41, 305 ff., trennt die worte mit entschiedenheit, und wir müssten alle umlernen, wenn er recht hat. Er geht von einem durchaus berechtigten standpunkt aus, und darin müssen wir ihm wol folgen, dass die altind. form bibhêti nicht urindisch ist, denn sie zeigt sich nur in jungen teilen des Rigveda, muss also als altindische sondererscheinung beurteilt werden. Nimmt nun Wackernagel in diesem falle einen specifisch indischen standpunkt ein, so dürfen wir wol auch dem ahd. bibên gegenüber einen specifisch deutschen und germanischen standpunkt vertreten. Betrachtet man die synonymen got. reiraiphs, ahd. bibêt und ahd. zittarôt (grundform titrôd), so springt der reduplicationstypus dieser wortgruppe in die augen (vgl. Beitr. 8, 342) und die genetische auffassung von ahd. bibêt als redupliciertes praesens bleibt bestehen. Dann kommt man natürlich doch auf eine idg. wurzel $b h i$; und wer wird die skepsis wol so weit treiben wie.Wackernagel, wenn er im beginn seines artikels sagt: „Diese etymologie ist falsch. Zunächst ist sie begrifflich wenig einleuchtend. In allen sprachen, wo ig. $b \hbar \bar{\imath}$ belegt ist, bedeutet es schlechtweg 'fürchten'. Wer es mit bibēn gleichsetzt, muss annehmen, entweder dass das germanische eine zugleich weitere und sinn- 
lichere bedeutung bewahrt habe, die auf indischem boden schon der veda ausgemerzt hätte und die auch iranisch, slavisch, litauisch mit seltsamer consequenz vermieden hätten, oder aber dass das verbum ursprünglich 'fürchten' bedeutet und man dann in germ. bebendes als 'sich fürchtend' bezeichnet habe. Ist eines oder das andere glaublich?"

Wenn wir worte der bedeutung 'beben' mit worten der bedeutung. 'sich fürchten' nicht mehr combinieren dürfen, dann müssen wir über-haupt unsere skepsis noch viel weiter treiben, als ängstliche gemüter wie ich sowieso schon tun.

Die hauptfrage für den etymologen ist und bleibt die forschung nach der wortverwantschaft. Aber wir sind nicht darüber einig, was wir unter der verwantschaft verstehen. Der eine versteht darunter einen genau präcisierbaren grad der familienzugehörigkeit, der andere meint eine nicht präcisierbare lose zugehörigkeit. Von einem specifisch deutschen standpunkt aus kann ich meinerseits viele sprachliche tatsachen der etymologie nicht als verwant ansehen, die von einem indogermanischen standpunkt aus verwant erscheinen. Die grenzen zwischen der wortgeschichtlichen betrachtung der einzelsprachen einerseits und den etymologischen versuchen der sprachvergleichung anderseits werden immer zu conflicten führen, und ich bin doch gewiss nicht das einzige beispiel dafür, dass der indogermanische standpunkt ein stärkeres betonen der entfernteren wortverwantschaften forderte. Dem wortforscher, der in den spuren Rudolf Hildebrands weiter voranschreitet, liegt es nahe, sich immer zu vergegenwärtigen, was im vorwort zum 5. bande des $\mathrm{DWb}$. über die etymologie gesagt wird: „Mir fällt es lange auf, dass gerade bei etymologischer arbeit die grundbegriffe von wirklich und möglich in ihrem scharfen unterschiede leichter und gefährlicher ins wanken geraten als bei jeder andern wissenschaftlichen arbeit, allenfalls die mythologische ausgenommen; wie vieles wird da mit einem 'ist' vorgetragen, oder mit 'ist gewiss' u. dgl., wo ein 'ist vielleicht, möglicherweise' v. dgl., höchstens ein 'ist wahrscheinlich' der wahrheit sich nähern würde."

Die etymologie zu einer exakten wissenschaft zu erheben, sind viele kräfte bemüht. Aber ebenso viele treiben noch das alte spiel mit vagen möglichkeiten, und wir wollen es getrost 
hinnehmen, dass unsere zeitschriften der tummelplatz aller möglichkeiten sind. Wir dürfen es auch ruhig geschehen lassen, dass specialwörterbücher, die nur fachleuten in die hand zu kommen pflegen, alle möglichkeiten, auch die unglaublichsten, verzeichnen und widergeben. Aber darf ein etymologisches wörterbuch der deutschen sprache, dessen leserkreis sich naturgemäss immer in weitere kreise erstreckt, verwirren und irreführen mit allen phantastereien und willkürlichkeiten, die innerhalb der fachwissenschaft ungefährlich wären? Ich habe oben an dem beispiel des neuen Weigand bewiesen, dass mein Etymologisches wörterbuch in bezug auf skepsis manchen neueren etymologien gegenüber keine sonderstellung einnimmt. Man vergleiche auch den dreibändigen Heyne und nun auch Pauls Deutsches wörterbuch, das in seiner 2. auflage auf etymologien nicht verzichtet. Wenn derartige hilfsmittel weitere kreise und das heranwachsende germanistengeschlecht von der ernsthaftigkeit sprachwissenschaftlicher arbeit überzeugen sollen, dann können wir nicht methodisch genug vorgehen, denn wir wollen den benutzer gewinnen und überzeugen, indem wir schritt für schritt voranschreiten. Die von Hildebrand charakterisierten luftsprünge der etymologen kann der deutsche wortforscher unmöglich mitmachen.

Aber eine schwierigkeit ist zuzugeben. Wer eine einzelne etymologie in unsern zeitschriften zu erörtern pflegt, oder auch eine kleinere wortgruppe etymologisch behandelt, ist in einer glücklicheren lage als der verfasser eines etymologischen wörterbuchs. Ueber jedes wort einer einzelnen sprache kann der lexicograph unmöglich mit derselben vorsicht und derselben exaktheit berichten, die ihm als ideal vorschwebt. Und sollen nicht auch unsere deutschen wörterbücher mit zusammenfassen, was andere vor und neben uns als sichere ergebnisse erzielt haben? Die lexicographen sind nicht allein schuld, dass noch nicht jedes wort sein etymon gefunden hat. Der neue Weigand wird den etymologen jetzt gewiss noch eben so viel zu tun übrig lassen, wie die 6. auflage meines Etymologischen wörterbuchs.

Auch die raumfrage pflegt bei den deutschen wörterbüchern eine rolle zu spielen. Die artikel meines Et. wb. z. b. können nicht den umfang annehmen, den einzelartikel aus dem 
bereich der etymologie in unseren zeitschriften haben. Nur ein ausschnitt aller vorgebrachten angaben und meinungen ist im rahmen eines kleinen artikels möglich. Gedrungenheit und knappheit gebietet sich ganz von selbst. Und so miissen ohne weiteres oft fernere oder unsichere verwantschaftsbeziehungen beiseite bleiben, wenn nähere verwantschaften ein wort hinlänglich aufklären. Wie gerne würde ich oft mit längerer erörterung in die debatte eingreifen, lieber würde ich manchmal einen grösseren zeitschriftenartikel schreiben und jedenfalls wäre es meist viel bequemer.

\section{III.}

Das wort braut (oben s. 557) hat eine reiche literatur um sich versammelt. Von den wörterbüchern abgesehen handeln darüber in speciellen aufsätzen: Torp, Sprvgl.-hist. studier tilegn. prof. C. R. Unger, s. 174; Uhlenbeck 1897, Beitr. 22, 188; Hirt 1897, Beitr. 22, 234; Wiedemann 1902, Bezz. Beitr. 27, 205-218; Loewe, Kuhns zs. 39,276; v. Domaszewski, N. Heidelb. jahrbücher 3, 193-198; Gundermann 1901, Zs.f.d.wortf. 1,240-246; Uhlenbeck 1905, Beitr. 30, 271; Braune, Beitr. 32, 30-59. 559-562. Wenn somit das wort auf etwa 100 seiten der verschiedensten zeitschriften erörtert worden ist, möchte der fernerstehende vermuten, dass alle wortgeschichtlichen fragen nunmehr definitiv erledigt wären, und doch hebt Braune, Beitr. 32, 53 und 57 die lückenhaftigkeit des wortgeschichtlichen wissens über braut mehrfach hervor. Ich bin nicht in der lage, sie zu ergänzen, glaube aber auch, dass die frage nach dem ursprung des wortes durch Braune nicht endgiltig gelöst worden ist. Er gestattet mir gerne, hier vorzubringen, was ich auf dem herzen habe und in der neuen auflage meines Et. wb. nicht mit der wünschenswerten ausführlichkeit vorbringen kann.

Den standpunkt, dass lat. Frutis als einzige etymologische möglichkeit in frage kommen kann, habe ich seit der 5. auflage meines Et. wb. vertreten und werde ihn auch in der 7. auflage genau so festhalten, obwol ich mir dafür den tadel Uhlenbecks (Beitr. 26, 292) zugezogen habe. Ich habe den gegenteiligen meinungen von Hirt, Torp, Uhlenbeck und Wiedemann nie widersprochen, weil meine lautgeschichtliche auffassung des wortes braut sich von anfang an bis heute gleich geblieben 
ist. Ich habe aber die gleichstellung mit lat. Frutis mit mehr vorsicht geäussert als Braune, und glaube die gleiche vorsicht noch jetzt walten lassen $z u$ müssen. Denn Braune hält den ansatz von $\hat{u}$ in Frutis für sicher auf die autorität von Waldes wörterbuch s. 249 hin. Aber sind dichterstellen und grammatikerzeugnisse für $\hat{\imath}$ beizubringen? Wer sich um die bei Georges und Forcellini gebotenen wortbelege bemüht, bemerkt bald, dass Waldes ansatz von $\hat{u}$ völlig aus der luft gegriffen ist. - Aber der göttin Frutis gegenübër ist auch sonst skepsis am platz. Wie mir Gundermann mitteilt, könnte an der oft citierten Festus-stelle, wo die handschriftliche überlieferung einen genetiv Fruti hat, möglicherweise Frutinis als gen. gestanden haben, und dann würde das eben dort erscheinende Frutinal ohne annahme von analogiebildung (vgl. Minerval neben Apollinar). leichter erklärbar sein. Üebrigens ist die mythologisch völlig dunkle Venus Frutis für die inhaltliche analyse des germ. wortes auch von Braune nicht ïberschätzt worden, denn sie spielt für die bestimmung der grundbedeutung von germ. braut auch bei ihm keine entscheidende rolle.

Wenn ich aber in bezug auf die verwantschaft mit lateinisch Frutis mit Braune annähernd einig bin, kann ich seiner behandlung der grundbedeutung des wortes nicht zustimmen. Bekanntlich vertritt Braune den standpunkt, dass 'das ursprüngliche bedeutungscentrum von brîd in der geschlechtlichen function des weibes làg (s. 45)': 'die ursprüngliche bedeutang des germanischen brîd definiere ich als » mulier quae cum viro concumbit« (s. 52).' Ich kann den beweis für diese annahme nicht für erbracht halten. Nach Braunes annahme müsste das wort eigentlich 'gattin' schlechtweg und nicht die 'neuver'mählte'. bezeichnen, und das lässt sich doch nicht erweisen. Dass mhd. briuten (s. 46). Braunes auffassung notwendig macht, bestreite ich auf grund von geheien, das Braune selber (s. 46) citiert. Die bedeutungsentwicklung beider worte ist so parallel, und man wird doch auch nicht darauf verfallẹn, dem urgerm. grundwort von mhd. gchîen dieselbe sexuelle grundbedeutung zu geben, die er für germ. brupis = brudis annimmt. An Braunes beweisführung habe ich hauptsächlich zu beanstanden, dass er die altgerm. zusammensetzungen mit braut nicht völlig wertet. Denn in den alten 
zusammensetzungen tritt nirgends eine sexuelle bedeutung zu tage, soidern nur eine rechtliche ('neuvermählte am hochzeitstage'). Zwar wird ags. brýd-ealo 'hochzeit' bei Murray erst ums jahr 1000 belegt, aber kann man ein höheres alter für altnord. brúd-hlaup und mndd. brutloft bezweifeln? Wenn darin eine altgerm. benennung der hochzeit liegt, so müssen wir darin das älteste zeugnis für die bedeutungsgeschichte unseres wortes anerkennen und wir kommen damit in eine zeit, die den belegen der römischen soldateninschriften bei Domaszewski und Gundermann eher voraufliegt als folgt.

Die grosse zahl der zusammensetzungen im ahd. und ags. ist Braunes auffassung keineswegs günstig. Man denke auch an ein völlig unerklärtes wort wie ags. brýtofta 'hochzeit', das in seiner dunkelheit den eindruck hohen alters macht. Ich sehe auch gar keine schwierigkeit ein, die rechtliche bedeutung als grundbedeutung an die spitze der wortgeschichte zu stellen. Wo in mhd. belegen eine sexuelle bedeutung vorliegt, kann dies doch wol als occasioneller euphemismus aufgefasst werden, der dann stereotyp geworden sein könnte. Das sind geläufige

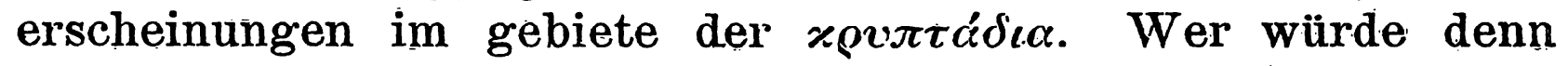
aus der spätmhd. bedeutung von minne einen schluss auf die grundbedeutung des wortes im 9.-12. jh. machen? Nach meiner meinung schafft Braune nur eine neue schwierigkeit, wenn er noch die bedeutung des jugendlichen alters für das grundwort mit in anspruch nimmt. Ich kann nirgends eine nötigung sehen, den begriff der jugendlichkeit in das grundwort zu verlegen. Aus der natur der sache könnte man das folgern, aber wie will man's in das wort hineinlegen? Nach dem DW. . IV 2, 2388 ist im nassauischen 'jungfrau' der name einer noch nicht lange verheirateten frau, daneben ehrenname der hausfrau, z. b. im munde der dienstboten, selbst wenn sie in hohem alter ist. Und Braune hat selbst eine ganze reihe von belegen aus der geschichte unseres wortes beigebracht, in denen ausschliesslich der rechtliche termin ohne rücksicht auf das lebensalter, aber vielleicht mit rücksicht auf die zeit vor der mutterschaft zum ausdruck kommt.

Während ich annehme, dass die sexuelle bedeutung nur secundär sein kann, lıat sich Braune bemüht, alle verschiedenen bedeutungsnuancen dem grundworte zuzutrauen und er begeht 
nach meiner meinung damit einen fehler, den man etymologen, wie z. b. mir selber, gelegentlich vorgeworfen hat. Nicht durch das addieren der verschiedenen bedeutungsnuancen kommen wir dem grundbegriff nahe. Ich möchte mir bei dieser gelegenheit erlauben, auch auf Braunes behandlung des wortes bett in diesen Beitr. 23, 250 (fussn.) einzugehen. Er knüpft dabei widerum an mein wörterbuch an: „Es ist nicht richtig, wenn Kluge im Etym. wb. (s. v. beet und bett) wegen des gartenbeets bett $\mathrm{zu}$ lat. fodio 'graben' stellt: die grundbedeutung von bett ist nur 'lager, lagerstatt, sitzstatt' gewesen. Diese bedeutung: hat sich schon in alter zeit besonders in der richtung 'polsterlager, polster' entwickelt. Aus der bedeutung 'lager, lagerstatt, sitzstatt' ist dann auch im westgermanischen die schon im ahd. und ags. vorhandene bedeutung 'standplatz von pflanzen, gartenbeet' hervorgegangen, wie sie im nhd. beet, engl. bed noch heute vorliegt." So elegant wie sich der neue Weigand in diesem falle aus der affäre zieht, bringe ich es leider nicht fertig. Mich plagen zu viel scrupel und zweifel. Aber eine blasse grundbedeutung 'standort' scheint mir weder für unser bett noch für unser beet zu genügen. Diese durch addition gewonnene grundbedeutung ist $\mathrm{zu}$ logisch, als dass wir sie an die spitze der wortgeschichte von beet, bett stellen können. Mit recht hebt Braune die schwierigkeit der wortgeschichtlichen überlieferung hervor: im ahd. tritt die bedeutung 'gartenbeet' relativ spät auf, im ags. die bedeutung 'standort für wildwachsende pflanzen' ebenso spät; die letzte fehlt dem ahd., aber die erste bedeutung ('gartenbeet') muss nach Braunes richtiger erwägung für den urzustand ausgeschaltet werden. Am liebsten möchte man mit Fischers Schwäb. wb. I 960 eine schlichte übertragung annehmen, so dass das gartenbeet von̉ seiner form benannt wäre. Aber wie verhält sich die ags. bedeutung 'standort der wildwachsenden pflanzen (binsen, schilf)' zu der ältesterreichbaren bedeutung des wortes bett, die sich auf grund der finnischen entlehnung als 'polsterkissen' vermuten lässt?

So ratlos wie in diesem falle bin ich dem worte braut gegenüber meinerseits vielleicht nicht: ich wage es, darüber eine entfernte möglichkeit hier mitzuteilen, die ich nicht für durchschlagend halte. Geht man von der allgemein anerkannten und nicht zu bezweifelnden analyse des femininums hlófaize 
neben dem zugehörigen masculin hláford im angelsächsischen aus - und niemand bezweifelt, dass in beiden worten ags. hláf 'brot' steckt - so darf ahd. brût an ahd. brôt erinnern. Nun tritt durch Braunes erörterung (Beitr. 32, 41, fussn.) ein ahd. proata mit der bedeutung 'nurus, bruta' in unsern gesichtskreis. Braune fasst das oa derselben als germ. $\hat{o}$. Aber aus seiner Ahd. gramm. $\S 45$, anm. 2 ergibt sich für die keronische glosse (Ahd. gll. I 216, 21) doch wol auch noch die möglichkeit, das oa nicht sowol für germ. $\hat{o}$ als für germ. au zu halten. Leider ist, wie Braune zeigt, die überlieferung des wortes sehr spärlich. Er vermutet ein schwaches femininum im obliquus als übersetzung des nominativs nurus, bruta und die von ihm beigebrachte spätahd. überlieferung brǒt als nominativ eines starken femininums lässt das möglich erscheinen, wenn man an das nachleben einer so singulären form bis in so späte zeit glauben könnte. Läge nicht vom standpunkt der wortbildungslehre eine suffixform wie in ahd. wirtun 'wirtin' nahe, das zwar nur bei Otfrid bezengt ist, aber gewiss höheres alter beansprucht? Darf man nun die stammsilbe des seltenen proatın auf brôt beziehen, so tritt die parallele mit ags. hlćfdize in ihr recht und wir würden nun die frage aufwerfen müssen: dürfte man dieses brôta (schw. fem.) 'nurus, bruta' wol von ahd. brût trennen, in dessen wortsippe die bedeutung 'nurus, bruta' eine so wichtige rolle spielt? Der äussere schein ist meiner vermutung günstig, aber nun kommen die formellen schwierigkeiten. Sie liegen einmal in dem worte brôt, dessen urgeschichte noch nicht definitiv aufgeklärt ist. Sie liegen auch in den forderungen der wortbildungslehre, die den charakter des ableitenden dentals gesichert haben will.. Ist der dental in brôt passivisch zu verstehen und in brût etwa persönlich? Oder sind ahd. brût und ahd. brôta gleichmässig als koseformen zu einem volltypus wie ags. hloefdize (eigtl. 'brotkneterin') zu verstehen? In betracht zu ziehen wäre, dass die etymologen ahd. brôt auch mit ahd. brod, engl. broth 'brühe' verbinden; und das verwante lat. defrutum 'eingekochter most', das mit der sippe von nhd. brauen sowie mit der hier behandelten wortgruppe in einem weiteren zusammenhange stehen soll, erinnert uns wider an das anklingende lat. Frutis. Nun scheint Braune dazu zu neigen, jenes proatun als 
schreibfehler für brût auffassen zu wollen, aber ich glaube kaum, dass man so die ganze von ihm behandelte glossengruppe aus der welt schaffen darf. Einerlei wie man sich dieser glossengruppe gegenüber verhaltén möge - die frage nach dem zusammenhang von ahd. brût mit ahd. brôt, von lat. defrutum mit lat. Frutis wird davon gar nicht betroffen. Und so mag mein verdacht hier seine stelle haben, denn einstweilen ist in meinem Etym. wörterbuch naturgemäss kein platz dafür: den eigenen etymologischen combinationen gegenüber zeige ich mich in meinem Et. wb. ebenso zurückhaltend, wie gegen unsichere vermutungen von mitforschern.

\section{IV.}

Vielleicht hat niemand mehr über etymologie auf dem. herzen, als ich selbst. Die zahllosen probleme, die in diesen Beiträgen zur debatte gestanden haben, beschäftigen mich nunmehr fast dreissig jahre, und ich werde nicht müde, an den schwierigkeiten des wortschatzes meine kräfte zu versuchen. Aber leider sehe ich viel zu oft die schwierigkeiten, die von andern mit stillschweigen übergangen werden. Wenn gedanken tatsachen wären, wären entschieden schon mehr untadelige etymologien zür verfügung, als es in wirklichkeit gibt. Aler hebel und schrauben genügen nicht immer, um der sprache ihre geheimnisse abzugewinnen. So hätte ich z. b. längst für ags. ides einen gedanken vortragen können, den das Beowulfepos nahelegt, wenn wir in seinem formelschatz eine ides Scyldinga (v. 1168) neben einem eodor Scyldinga (v.428. 663) antreffen: den naheliegenden gedanken, dass eodor als masculinum zu ides als femininum gehört oder umgekehrt, habe ich mich gehütet $\mathrm{zu}$ veröffentlichen, weil ich nicht in der lage bin, die oben s. 556 f. behandelten schwierigkeiten mit dieser neuen deutung in einklang zu bringen. Das wort macht viel zu viel schwierigkeiten vom standpunkt der lautlehre, formenlehre und wortbildungslehre, als dass mich irgendeine bisher vorgetragene erklärung befriedigte. Und meinen gedanken habe ich hier nur zum besten geben wollen, um zu zeigen; dass es noch immer möglichkeiten einer deutung gibt, wenn man es mit den formellen schwierigkeiten nicht ernst nimmt. Aehnlich bin ich verfahren, als Leitzmann vor zwei 
jahren in diesen Beiträgen 32,60 eine neue, allerdings formell nicht stichhaltige erklärung von ags. neorx-nawonz vortrug. Ich zeigte alsbald in der Zeitschrift $f$. deutsche wortforschung 8,144 , dass es noch immer neue möglichkeiten der deutung gibt. Und doch bin ich keineswegs von der richtigkeit meiner deutung des'schwierigen wortes überzeugt: ich wollte nur ein experiment der wortanalyse machen. Und so bin ich auch weit davon entfernt, meine annahme von verwantschaft $z$ wischen ahd. brût und ahd. brôt als sicher auszugeben: ich wollte nur zeigen, dass unsere eifrigen etymologen, die jetzt am werke sind, noch immer nicht alle sprachlichen möglichkeiten erschöpft haben, so viel über brût auch schon geschrieben ist. Ich habe mich bisher aus meiner grammatischen position nicht verdrängen lassen und etymologien nur dann veröffentlicht, wenn ich der überzeugung war, alle intern germanischen schwierigkeiten grammatisch lösen zu können. Nur wenn lautlehre, formenlehre und wortbildungslehre mit der betreffenden wortbedeutung in einklang standen, habe ich neue etymologien vorzutragen gewagt. Manche präliminarische gedanken habe ich lieber für mich behalten, als unfertiges, unglaubliches vorzutragen.

Je mehr wir die sprachlichen tatsachen vernachlässigen, um so schneller sind wir mit etymologien fertig. Das sieht man beispielsweise auch an Feists behandlung des wortes köter, die mir schon vor ihrem abdruck in diesen Beiträgen $(33,402)$ bekannt geworden war. Er setzt für das ndd. köter zusammenhang mit dem rheinfr. kauzen, gauzen 'bellen' voraus, wofür er eine grundform *kutjan annimmt. Wir erfahren aber nichts darüber, warum der umlaut fehlt; nichts darüber, dass im Grimmschen wörterbuch unter gauzen der gleiche verbalstamm mit anlautendem $g$ für den ganzen Oberrhein und auch sonst weithin bezeugt ist, nichts darüber, dass in einem verbum gauzen naturgemäss das bekannte secundärsuffix got. -atjan = ahd. -azzen stecken muss, da in einem. schallwort das intensivsuffix wahrscheinlich ist. Und wir erfahren auch nichts darüber, dass eine secundärbildung liöter als 'beller' gerade für Niederdeutschland ein solches schallwort 'bellen' voraussetzt, wo Feist es leider nicht nachgewiesen hat. Schliesslich aber nuitzt uns ein hypothetisches verbum kütjan gar nichts, um 
eine secundärbildung altsächs. *kôteri zu erklären: die vocale stimmen ja gar nicht! Wen sprachliche erwägungen dieser art nicht quälen, der kann uns noch mit vielen etymologien überraschen. Wer es aber mit den forderungen der lautlehre und den gesetzen der wortbildung ernster nimmt, hat da einen schweren stand. Er erweckt deñ verdacht, dass er zurückgeblieben ist, wenn er den tollen sprüngen nicht folgen kann. Auf keinem gebiete unserèr wissenschaft sind mehr steine aus dem wege zu schaffen, mehr schutt zu beseitigen, mehr grobe verstösse anzutreffen, als auf dem gebiete der etymologie. Freilich ist es ein unerfreuliches geschäft, sich dèrartiger rein negativer arbeit zu unterziehen, man unternimmt eine derartige kritik nur dann gern, wenn man selber einen definitiven schritt vorangekommen ist, wie es Kluyver in seinem artikel tolpatsch (Beitr. 30, 211) getan hat. Er hat sich nicht irre machen lassen durch Schröders apodiktische behauptung, die mit den worten vorgetragen wird: 'es darf daher als sicher angenommen werden ...' (Beitr. 29, 558). Und wenn Schröder (Beitr. 29, 559) eine von meiner darstellung abweichende erklärung des wortes Finkeljochen mit den worten einleitet: 'das ganze wort erklärt sich sehr einfach', so darf ich auch hier wider den neuen Weigand als meinen bundesgenossen beschwören, der Finkeljochen in übereinstimmung mit mir als rotwelsch charakterisiert ohne Schröders neue deutung zu verwerten. Ich müsste hier meine rotwelschen sammlungen vorführen, wenn ein gewissenhafter arbeiter nicht aus meinem Rotwelschen quellenbuch sich das belegmaterial leicht beschaffen könnte:

Machen aber schon die intern germanischen schwierigkeiten den deutschen etymologen unliebsam viel zu schaffen - wie kann dieser dann noch der endlosen schwierigkeiten herr werden, die uns im albanesischen und armenischen, im kurdischen und pehlevi entgegentreten? Behauptungen der art, dass ein deutsches wort seine nächsten verwanten auf dem oder jenem schwer controllierbaren gebiet hätte, müssien jedem forscher misbehagen machen, der gelernt hat, sich mit den schwierigkeiten eines engeren sprachgebiets abzufinden. Aber wie gross sind nicht auch schon die schwierigkeiten auf den durchsichtigeren und eher controllierbaren sprachgebieten des griechischen und lateinischen! Der germanist, der sich mit 
den deutschen mundarten und den literarischen zeugnissen der vergangenheit abfindet, kann in die italische und griechische dialektkunde und wortgeschichte nicht ungestraft übergreifen. Wie leicht wäre es, zu behaupten, dass ahd. warnen, ags. wyrnan

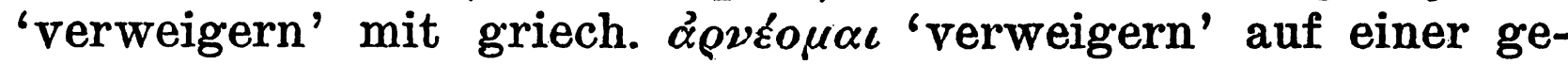
meinsamen grundform ruhte, und doch hat griech. ảovéouaı aller wahrscheinlichkeit nach im anlaut kein $f$ verloren. -

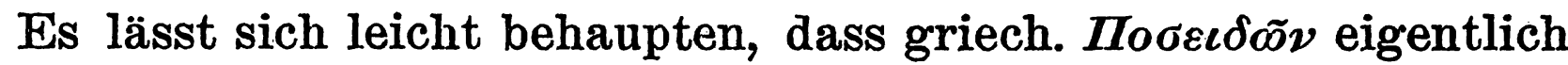
vocativform $\mathrm{zu}$ einem $\pi \delta$ ó $\iota s$ $2 \alpha \tilde{\tilde{\nu} \nu}$ gewesen wäre, aber mit einem solchen gedanken wäre niemandem gedient, so lange nicht alle schwierigkeiten, die das griech. wort macht, mit einer neuen deutung in wegfall kommen. - Für griech. $\delta \varepsilon v \dot{v} \varepsilon \varrho o s$ läge von seiten der: bedeutung nichts näher, als eine griech. grundf. * $\delta \varepsilon F o \tau \varepsilon \rho o \varsigma$, die dreisilbig geworden wäre wie qi

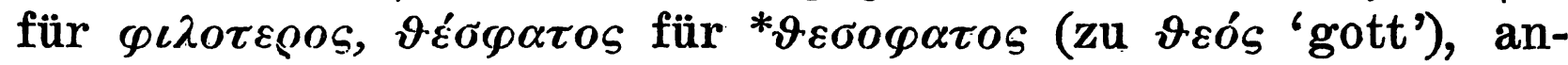
zunehmen und $* \delta \varepsilon F o=\delta v o$ 'zwei' zu setzen. Derartige etymologische combinationen, wie sie im bereich jener vagen möglichkeiten liegen, die man heute bei so vielen etymologen trifft, muss man sich hüten drucken zu lassen, denn es bleiben immer nur präliminarische gedanken vorbereitender, heuristischer art, aber keine wissenschaftlichen ergebnisse.

Naturgemäss genügen aber dem etymologen die historisch documentierbaren sprachlichen tatsachen nicht und ohne hypothesen, ohne hypothetische grundformen ist etymologie undenkbar, aber das ergänzen der zwischenglieder bleibt immer nur hypothese ohne gewähr. Wo wir alle mit verschiedenen, von einander abweichenden voraussetzungen operieren, bleibt auch die abwägung der wahrscheinlichkeit immer nur subjectiv.

Ich habe es schon lange für möglich gehalten, dass das romanische wort für blond ein altgermanisches fremdwort ist; aber das germ. blunda ist in keiner germanischen sprache anzutreffen. Nun stellt Nigra (Romania 26,555) für das roman. wort eine neue lateinische etymologie auf: er setzt ein hypothetisches albundus voraus und lässt dies über vulgärlat. ablundus zu blundus werden. Ich weiss nicht, wie die romanisten sich $\mathrm{zu}$ dieser neuen theorie verhalten. Müsste aber nicht eine derartige hypothetische bildung des vulgärlateins vielmehr albicundus heissen, wie es rubicundus heisst? Ist eine umstellung von albundus $\mathrm{zu}$ ablundus wahrscheinlich?

Beiträge zur geschichte der deutschen sprache. $\mathrm{X} \times \mathrm{XV}$. 
Von solchen schwierigkeiten sehe ich ganz ab und frage nur, ist der ansatz eines hypothetischen wortes für das latein etwa leichter als für das germanische? Das latein, in dem sich das vulgärlatein widerspiegelt, ist für die mutmassliche periode der entlehnung umfassend documentiert, aber das germanische der völkerwanderungszeit hat im wesentlichen nur Ulfilas zum zeugen. Keine germanische sprache kennt das framea der antiken überlieferung in derselben bedeutung, und doch bezweifelt niemand den germanischen charakter des wortes. Die wahrscheinlichkeit, dass ein altgerm. blundus 'blond' bestanden hat, scheint mir immer noch wahrscheinlicher als jenes hypothetische lat. albundus.

Wo mit statistik nicht operiert werden kann, ist exakte beweisbarkeit immer fragwürdïg. Immer von neuem wider werden hypothesen zu controversen führen. Ich habe mich nicht gescheut, hier einmal bedenken zur sprache zu bringen, die mich schon lange beschäftigen. Sie werden zeigen, dass schweigen nicht immer zustimmung bedeutet. Und ich habe vielfach schon zu lange geschwiegen, weil ich darnach trachtete, durch beschäftigung mit den standessprachen der deutschen etymologie den boden zu befestigen.

Denn die deutsche etymologie hat sich nicht bloss in den nebel der idg. urzeit vorzuwagen. Der indogermanist ist gar keine competente instanz über ein etymologisches wörterbuch der deutschen sprache. Dafür bietet die interne sprachbetrachtung von einem ausschliesslich germanischen und von einem ausschliesslich deutschen standpunkt doch sicher die übermasse der probleme. Und vieles muss rein intern germanisch gedeutet werden, wo der indogermanist ganz vergebens sich abmüht. Als Möller, Kuhns zs. 24, 460 für ags. fracoð 'verachtet' eine latein. verwantschaft suchte und $\mathrm{zu}$ finden glaubte; konnte ich (ebda. 26,72 ) das wort auf grund von ags. unforcuð als rein germ. wortschöpfung analysieren. Unter die intern germanischen probleme, die der beurteilung des indogermanisten gar nicht unterstehen, gehört auch die feststellung allen lehnmaterials: wenn z.b. nhd. kerze aus lat. carta oder engl. taper aus lat. papyrus als lehnworte gedeutet werden, so werden das nur etymologen aus dem bereiche der indogermanistik werten können, die daraus lernen wollen, dass für nhd. kerze und engl. taper keinerlei 
indogermanistische deutungen vonnöten sind. Dazu kommen die zahllosen ermittelungen, die der deutsche etymologe über geographische oder chronologische wortbestimmungen beschaffen muss. Wer für bittsteller oder kerbtier den bekannten puristen Campe als wortschöpfer ermittelt, darf sich als etymologe vielleicht ebenso viel einbilden, wie Uhlenbeck über seine verwantschaft von ags. ides 'vornehme frau' mit skr. atasa 'gebüsch'. Aber wird es der indogermanist in dem bereich seiner studien auch anerkennen oder würdigen können, wenn ein etymologisches wörterbuch der deutschen sprache bittsteller oder kerbtier dem ursprunge nach aufklärt?

Wenn nun eine grosse kluft den deutschen wortforscher nicht immer, aber fast immer von dem indogermanistischen etymologen à la Uhlenbeck trennt, darf er sich doch oft der stillen, meist allerdings nicht verlautbarten zustimmung ernsterer indogermanisten erfreuen. Aber viel wichtiger als stille zustimmungen $\mathrm{zu}$ meinen etymologien war mir eine äusserung Pedersens über die wissenschaftliche stellung meines Etymol. wörterbuchs der deutschen sprache, wenn er 1904 im Arkiv för Nordisk Filologi 20, 383 sagt, dass Falk und Torp als verfasser des Etymologisk Ordbog over det norske og det danske Sprog den gleichen orakelglauben meinem wörterbuch gegenüber hegen, wie ich selber.

FREIBURG i. B.

F. KLUGE.

\section{ZU PSALM 138.}

V. 8 der ahd. metrischen bearbeitung von Psalm 138 (nach der hier noch übereinstimmenden zählung der Denkmäler und von Braunes Lesebuch ${ }^{6}$ ) lautet: Den uuech furiunorhtostu mir, daz ih mih cherte after dir. Der sinn der stelle ist bestritten, aber man kommt doch immer wider darauf zurück, das verbum furiwurchen als 'vorher bereiten', 'praeparare' zu erklären (s. z. b. MSD 23,87 und Braune a.a.o. im glossar), und zwar offenbar in mehr oder weniger bewusster anknüpfung an das praevidisti des lat. textes, das doch schon in z. 6 seinen ausdruck gefunden hatte. Dass das aber eigentlich nicht angeht, 